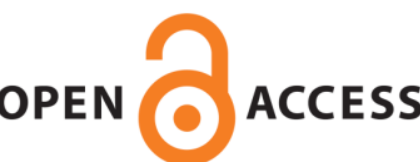

UWS Academic Portal

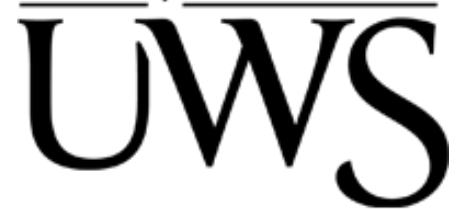

\title{
Enhanced performance and hindered membrane fouling for the treatment of coal chemical industry wastewater using a novel membrane electro-bioreactor with intermittent direct current
}

Hou, Baolin; Kuang, Yu; Han, Hongjun; Liu, Ye; Ren, Bozhi; Deng, Renjian; Hursthouse, Andrew S.

Published in:

Bioresource Technology

DOI:

10.1016/j.biortech.2018.09.063

Published: 31/01/2019

Document Version

Peer reviewed version

Link to publication on the UWS Academic Portal

Citation for published version (APA):

Hou, B., Kuang, Y., Han, H., Liu, Y., Ren, B., Deng, R., \& Hursthouse, A. S. (2019). Enhanced performance and hindered membrane fouling for the treatment of coal chemical industry wastewater using a novel membrane electro-bioreactor with intermittent direct current. Bioresource Technology, 271, 332-339.

https://doi.org/10.1016/j.biortech.2018.09.063

\section{General rights}

Copyright and moral rights for the publications made accessible in the UWS Academic Portal are retained by the authors and/or other copyright owners and it is a condition of accessing publications that users recognise and abide by the legal requirements associated with these rights. 
1 Enhanced performance and hindered membrane fouling for the treatment of coal

2 chemical industry wastewater using a novel membrane electro-bioreactor with

\section{3 intermittent direct current}

4 Baolin $\mathrm{Hou}^{\mathrm{a}, *}$, Yu Kuang ${ }^{\mathrm{a}}$, Hongjun Han ${ }^{\mathrm{b}}$, Ye Liu ${ }^{\mathrm{a}}$, Bozhi Ren ${ }^{\mathrm{a}}$, Renjian Denga ${ }^{\mathrm{a}}$, Andrew S

5 Hursthouse $e^{\mathrm{a}, \mathrm{c}}$

6 a. Hunan Provincial Key Laboratory of Shale Gas Resource Utilization, School of Civil

7 Engineering, Hunan University of Science and Technology, Xiangtan 411201, China

b. State Key Laboratory of Urban Water Resource and Environment, Harbin Institute of

Technology, Harbin 150090, China

c. School of Science \& Sport, University of the West of Scotland, Paisley PA1 2BE, UK

Abstract: A membrane electro-bioreactor (MEBR) embracing biological treatment,

electrokinetic phenomena and membrane filtration was established by applying intermittent direct current (DC) to MBR. MEBR exhibited significant improvement of treatment performance and reduction of membrane fouling. COD and total phenols removal efficiencies increased to $83.53 \%$ and $93.28 \%$ at an exposure mode of $24^{\prime}-\mathrm{OFF} / 6^{\prime}-\mathrm{ON}$, compared to $71.24 \%$ and $82.43 \%$ in MBR. Trans-membrane pressure increment rate declined dramatically in MEBR, which was mainly attributed to the increase of sludge floc size and decrease of zeta potential, soluble microbial products and specific resistance to filtration, resulted from electrokinetic effects such as electrocoagulation, electrophoresis, electroosmosis and electromigration of ions. It was notable that DC exposure exerted distinct evolution on microbial community, with the improvement of microbial community richness and diversity. The relative abundances of functional genera were promoted noticeably in MEBR. An interactive relevance existed among microbial community structure, mixed liquor properties 
and operational parameters.

Keywords: membrane electro-bioreactor; membrane fouling; electrokinetics; mixed liquor properties; microbial community; relationship analysis

\section{Introduction}

New coal chemical industry has been rapidly booming in China due to the stresses of environmental pollution and energy shortage according to the national energy development strategy, which is a comprehensive utilization of coal resource in a cleaner way. However, the coal gasification process, an indispensable procedure in coal chemical industry, discharges enormous amount of high-strength wastewater containing various of toxic compounds and a great number of refractory organic and inorganic pollutants such as phenolic compounds (main organic pollutants), nitrogen-containing heterocyclic compounds (NHCs), polycyclic aromatic hydrocarbons (PAHs), long chain hydrocarbons, ammonia, cyanide and thiocyanate et al (Xu et al., 2016). The high-strength wastewater has posed great pollution risk to ecological environment due to its toxicity and refractory, especially without appropriate treatment (Hou et al., 2015; Xu et al., 2018). Membrane bioreactor (MBR) integrates activated sludge process with membrane filtration and has been applied to the treatment of domestic wastewater and various kinds of industrial wastewater as well as wastewater reuse with the advantages of higher mixed liquor suspended solids (MLSS) and organic pollutant loading, superior effluent quality, independent control of hydraulic retention time (HRT) and solids/sludge retention time (SRT) et al (Barreto et al., 2017; Chen et al., 2017). The conventional MBR refers to aerobic MBR. With the development of MBR technology, anaerobic MBR (AnMBR) emerged for the complement of membrane biological system. AnMBR allows energy recovery by conversion of organic matter into methane-rich biogas 
during the anaerobic digestion(Martin Garcia et al., 2013; Ruigómez et al., 2017). Both types of MBR have been considered as the preferential available technology with potential application in water and wastewater treatment(Huang and Lee, 2015; Xie et al., 2016; Liu et al., 2018). However, membrane fouling remains the major issue to be solved to ensure the sustainability of this promising technology(Ahmed et al., 2018; Fan et al., 2018). The reduction of flux and rise of trans-membrane pressure (TMP) caused by membrane fouling resulted in the increase of operational costs and deterioration of effluent quality(Jia et al., 2014; Meng et al., 2017). Generally, membrane fouling was classified as reversible (recoverable by physical methods) and irreversible (including irreversible can be removed through maintenance cleaning, irreversible that can be removed through chemical cleaning and irrecoverable fouling) (Ibeid et al., 2013). Factors affecting membrane fouling include membrane properties, influent and sludge characteristics (soluble microbial products (SMP), floc size, colloids et al.) as well as operational conditions (MLSS, SRT, HRT et al.) (Tian et al., 2011). Several approaches had been attempted to hinder membrane fouling (Iorhemen et al., 2017). However, it can be concluded from the attempts that searching for an effective and powerful method to reduce membrane fouling at an acceptable range remains a formidable challenge. Recently DC was introduced to MBR for the enhancement of pollutant removal and fouling reduction (Bani-Melhem and Elektorowicz, 2011; Jiang et al., 2017). Synergic effects worked on the treatment processes in the integrated system. Electrodes were placed in MBR to form electric field as well as involve electrochemical reactions. The activity of most microbes could be promoted by adequate electric field stimulation, leading to the enhancement of treatment performance. Small-size soluble and colloid components were reduced by electrochemical effects to migrate membrane fouling. Some researchers inserted a 
cathode inside the membrane to form electric field with an anode outside the membrane module, in which the negatively charged extracellular polymeric substance (EPS) were forced to move away from the membrane surface and then effectively attenuated the precipitation of EPS on membrane surface and membrane fouling(Liu et al., 2012). However, this membrane module with built-in cathode was complex and constrained by the module form and adversely affected the integrity of membrane module. In addition, few studies focused on the treatment of industrial wastewater and microbial community dynamics induced by DC in the system were rarely conducted. The relationships among intermittent DC mode, properties of mixed liquor and microbial community dynamics were not clear.

In the current study, membrane electro-bioreactor (MEBR) coupling of MBR and intermittent DC with iron anode was constructed to treat coal chemical industry wastewater. The primary objective of this research was to evaluate the performance of efficiency enhancement and fouling reduction. The variations of sludge mixture properties (such as zeta potential, floc size, SMP, specific resistance to filtration (SRF) et al.) were inspected to analyze the possible mechanism in MEBR. The microbial community in MEBR was investigated preliminary by the high-throughput sequencing technology. Moreover, the relationships among intermittent DC mode, current density, properties of mixed liquor, membrane fouling and microbial community dynamics were analyzed, which was conductive to a deeper understanding of the mechanism and optimization control.

\section{Materials and methods}

\subsection{Materials}

Real coal chemical industry wastewater was obtained from the full-scale wastewater treatment facility of a coal chemical industry plant in China, and the wastewater samples used 
in this study were the effluent of anaerobic treatment process. The wastewater characteristics were as follows: COD $1486.4 \pm 102.4 \mathrm{mg} / \mathrm{L}, \mathrm{BOD}_{5} 253.3 \pm 18.2 \mathrm{mg} / \mathrm{L}$, total phenols $233.8 \pm 21.2$ $\mathrm{mg} / \mathrm{L}$, TOC $335.6 \pm 22.3 \mathrm{mg} / \mathrm{L}, \mathrm{NH}_{4}-\mathrm{N} 127.2 \pm 8.5 \mathrm{mg} / \mathrm{L}$. The inoculum sludge was collected from the A/O tank, with SVI of 88.

\subsection{Experimental procedure}

A cylindrical Plexiglas bioreactor with the volume of $3.0 \mathrm{~L}$ was used as the reaction medium. A hollow fiber membrane module (normal pore size $0.4 \mu \mathrm{m}$, effective surface area $0.02 \mathrm{~m}^{2}$ ) was positioned at the center of reactor to construct MBR. In MEBR, stainless steel plate and graphite plate were installed inside the bioreactor as anode and cathode. Both electrodes with the same dimension of $3.0 \times 3.0 \mathrm{~cm}$ were fixed on two plastic brackets with the distance of $10 \mathrm{~cm}$. The electrodes were connected with a DC power supply which was controlled by a timer to achieve intermittent DC. Current density was maintained at 1.33 $\mathrm{mA} / \mathrm{cm}^{2}$. Air was bubbled from diffuser at the bottom of the reactor to supply oxygen and shear stresses on the membrane surface. The effluent from the membrane module was withdrawn via a peristaltic pump at the mode of suction 9.0 min followed by a $1.0 \mathrm{~min}$ rest. HRT was maintained at 18 hours. The bioreactors were operated at complete SRT, which was to say, no sludge was discharged from the bioreactors during the whole process except the samplings for the properties measurements of mixed liquor. A MEBR and a MBR (controlled trials) were operated simultaneously. Electrical exposure mode was defined as the ratio of time without current to time with current in a cycle of $30 \mathrm{~min}$. In the trials for relationship analysis, current density varied in the range of $0.5-1.33 \mathrm{~mA} / \mathrm{cm}^{2}$, and exposure mode ranged in $2-5$.

\subsection{Analytical methods}



diffraction particle size analyzer. Zeta potential was measured with the Zeta Sizer 3000 analyzer. The specific oxygen uptake rate (SOUR) was calculated from the linear regression slope of the DO drop vs. time divided by the VSS concentration in the test chamber (Hou et al., 2014). SRF was calculated with the Carman equation (Formula S1) based on the data of filtrate volume versus time in vacuum filtration test. Proteins and polysaccharides contents in SMP were measured calorimetrically following Dubois et al. (Dubois et al., 1956) and Lowry et al. (Lowry et al., 1951) methods. Microbial community was analyzed via high-throughput sequencing technology using Miseq Illumina followed by DNA extraction and PCR according to the methods in the literatures (Ma et al., 2015; Jia et al., 2016). Membrane fouling rate was membrane module was disassembled from the reactor and cleaned thoroughly with tap water COD and total phenols removal efficiencies in MEBR at mode 4 increased to $83.53 \%$ and 
62.61\% and 73.19\%. Enhancement of DC declined with lower electrical exposure (mode 5, $77.16 \%$ for COD, $89.58 \%$ for total phenols). Appropriate electrical exposure promoted treatment performance, while excess electrical exposure (mode 2) constrained the effectiveness. Large amounts of iron ion were generated when MEBR run at high electrical exposure, and high concentration and precipitation of iron ion inhibited the activity of microbes in the sludge, thus exerting adverse effect on the treatment performance. Moreover, it was found that pollutant removal efficiency in MEBR exceeded the sum of removal efficiency by MBR and that by single electrochemical effect (only electric field worked). For example, the sum of COD and total phenols removal efficiencies by MBR and single electrochemical effect were $73.62 \%(71.24 \%+2.38 \%)$ and $86.21 \%(82.43 \%+3.78 \%)$, lower than that in MEBR at mode 4. The comparison implied that a synergic effect existed between biodegradation and electrochemical effect in MEBR. Performance improvement at appreciate electrical exposure mainly resulted from the changes of the mixed liquor and microbial community in activated sludge. Therefore, changes in the properties of the mixed liquor and microbial community were discussed in the subsequent section. Mode 4 was selected to conduct the following tests as comprehensive consideration of performance, energy consumption as well as electrode dissolution. In addition, less foam was observed in MEBR, which prevented the biomass from reducing due to dehydration of sludge caused by foam, guaranteeing the high treatment performance.

Organic compositions in the wastewater were detected via GC-MS (Table 1). The contents of various organic compounds decreased in the effluents of MBR and MEBR. Phenolic compounds were nearly completely removed, which was consistent with the high removal efficiencies both in MBR and MEBR. Long-chain hydrocarbons and part of 
nitrogenous heterocyclic compounds were residual in the effluent. Moreover, the contents and number of main organic compounds in the effluent of MEBR were less than that in the effluent of MBR, which was probably attributed to the synergic effect of MBR and electrochemical effects and the variation of microbial community in MEBR. This also verified the enhancement efficiency of MEBR.

\subsection{Membrane fouling}

TMP variation with operation duration in MBR and MEBR with MLSS of $4500 \mathrm{mg} / \mathrm{L}$ was depicted in Fig. 2a. The increase of TMP in MBR and MEBR was similar at the first 25 days. However, the first washing time in MBR was the 33rd day, and that in MEBR delayed to the 47 th day. The membrane in the MBR was cleaned 3 times compared to only 1 time for MEBR. Membrane fouling rates (expressed by TMP increment over time) in MBR during each membrane washing were $0.23 \mathrm{kPa} / \mathrm{d}$ and $0.55 \mathrm{kPa} / \mathrm{d}$, and that in MEBR was $0.20 \mathrm{kPa} / \mathrm{d}$, exhibiting significant fouling reduction with the addition of intermittent DC. The comparison of TMP variation demonstrated that membrane fouling can be recovered better via physical cleaning in MEBR than MBR. This is mainly attributed to the effect of intermittent DC on microbes and properties of the mixed liquor, which lowered the contents of fouling compounds (SMP, colloidal materials, VSS et al.) contributing to irreversible membrane fouling. Moreover, TMP variation in MBR and MEBR with MLSS of $3000 \mathrm{mg} / \mathrm{L}$ was also investigated (Fig. 2b). Membrane washing in MBR was 2 times compared 1 time for MEBR. The comparison of different MLSS suggested that membrane fouling reduction by MEBR with high MLSS (high fouling potential) was more significant than that with low MLSS (low fouling potential). The superiority of MEBR was less pronounced when the sludge had a low fouling potential, though noticeable. 


\subsection{Changes in the properties of the mixed liquor}

Performance improvement and membrane fouling reduction in MEBR were associated with significant changes in the properties of the mixed liquor. It was reported that SMP, sludge floc size, SRF were the key components determining the sludge fouling potential(Ibeid, Elektorowicz et al., 2013). The changes in the properties of the mixed liquor were analyzed, which provide a quantifiable basis for estimating membrane fouling.

Zeta potential determines the capability of microbial flocs to aggregate. The zeta potential of flocs in MBR increased slightly during the operation (average value from -17.6 $\mathrm{mV}$ to $-24.7 \mathrm{mV}$ ), while a significant zeta potential reduction was observed in MEBR (Fig. 3a). The average value decreased to $-12.1 \mathrm{mV}$ in MEBR, which was attributed to the Fe ions released from the anode via electrolysis. Fe ions neutralized the negative charge on the solid surfaces and microbial flocs and thereby reduced the repulsive forces between the flocs. $\mathrm{Fe}$ ions led to the coagulation of colloidal particles and sludge flocs. This change of zeta potential reflected the possibility of bio-flocculation and the formation of larger floc size, as discussed subsequently.

Floc PSD changed in a way that reflected the impact of DC field on floc morphology and structure. Fig. $3 b$ illustrated the PSD variations in MBR and MEBR. Floc PSD in MBR maintained at $123.6 \mu \mathrm{m}$. In contrast, floc PSD increased from $96.6 \mu \mathrm{m}$ to nearly $157.1 \mu \mathrm{m}$, and then decreased and stabilized at $134.1 \mu \mathrm{m}$. The initial increase was caused by the electrocoagulation due to the suppression of diffuse double layer as the reduction in zeta potential, during which organic particles formed larger flocs and colloidal solids were reduced. The increase of floc PSD was also corroborated by enhanced movement of the charged solid particles as a result of applying an electrical field. Subsequently floc size recession was 
associated with the electroosmotic extraction of the tightly bound water from the flocs.

Furthermore, the sludge in MEBR with a higher percentage of inorganic solids was likely to have less electrostatic attraction forces with bound water. Moreover, ferric hydroxides flocs or flocs combined with other solids with smaller size shifted the floc size distribution toward lower values. The floc size was likely to stabilize at a certain value based on the average size of the hydroxide complexes and electrical density. The increase in floc size was widely known to hinder membrane fouling (Tafti et al., 2015). Small particles depositing on the membrane surface or inside the voids of the cake layer caused an increased membrane resistance. The bound water within the sludge flocs was one main sludge property affecting its fouling potential. The change in floc size increased floc PSD and declined bound water, treatment performance. thus reduced membrane fouling significantly. In addition, sludge with large floc size, high density was kept from being taken away with foam, guaranteeing the high biomass and

It was evident that a significant reduction of SMP was observed in MEBR compared to MBR (Fig. 3c). SMP in MEBR and MBR at 50th day were 29.24 and $51.37 \mathrm{mg} / \mathrm{L}$ respectively. SMP was removed as a result of electrokinetic effects through which the ferric cations neutralized the negatively charged particles. Proteins with high density of negative charge reacted electrostatically with the ferric cations to form stable flocs. SMP was considered as a major component causing membrane fouling (Tian, Chen et al., 2011). Electrokinetic effects in MEBR had considerable contribution to the removal of SMP, which made the sludge and the cake layer on membrane less viscous and cohesive. Thus, the filterability of sludge was improved and membrane fouling was reduced.

A substantial reduction of SRF in MEBR was observed compared to that in MBR (Fig. 
3d). SRF of mixed liquor in MBR was around $3.52 \times 10^{13} \mathrm{~m} / \mathrm{kg}$, while that in MEBR stabilized at $1.96 \times 10^{13} \mathrm{~m} / \mathrm{kg}$. The reduction of SRF was mainly related to the changes of floc size, SMP, flocs bound water resulted from electrokinetic effects such as electrocoagulation, electrophoretic, electroosmosis, ions electromigration et al. SRF was in inverse proportion to the square of particle diameter according to Carman-Kozeny equation (Bani-Melhem and Elektorowicz, 2010). The increase in floc size caused by electrocoagulation in MEBR declined the SRF and minimized membrane fouling. In addition, the reduction of MLVSS/MLSS (from 0.8 to around 0.68) in MEBR also exerted positive effect on attenuating SRF. The reduction of SRF facilitated the following processes for excess sludge treatment. The reduction of MLVSS/MLSS was mainly related to electrocoagulation by the electrolysis on the iron anode. Iron content of the sludge in MEBR was around 5\%, compared to that of $0.35 \%$ in MBR. The iron flocs changed the water distribution in the sludge, thus improved the dewaterability. The component of easily dewatered iron floc also facilitated the decline of SRF and promotion of dewaterability.

SOUR was a major indicator of microbial activity. Microbial activity expressed by SOUR of activated sludge in MEBR gone up to $12.53 \mathrm{mgO}_{2} /(\mathrm{g}$ MLVSS $\cdot \mathrm{h})$ compared to that of $10.68 \mathrm{mgO}_{2} /(\mathrm{g}$ MLVSS·h) (Fig. S1), showing an appreciable increase after applying intermittent DC. Though the sludge was a rather complex composition, SOUR could be considered as an indication of maintaining high microbial activity in MEBR. At an appreciate current density and exposure, the improved microbial activity in MEBR translated into amenability to application as a bioelectrical reactor where DC stimulated microbial metabolism and/or pure cultures (Thrash and Coates, 2008).

\subsection{Microbial community}



microbial activity and promoting microbial community. High throughput sequencing was introduced to investigate the microbial community richness, diversity and structure shift in MBR and MEBR. Some differences of microbial community were observed between MBR and MEBR. Microbial community richness and microbial diversity were both promoted in MEBR as indicate by Chao 1 index and Shannon index (Fig. S2). It was assumed that the differences in microbial community richness and diversity were related closely to the addition of intermittent DC. The larger size and compact sludge floc in MEBR provided a new growth environment, which supplied a core carrier for the growth and propagation of microbes(Xin et al., 2016; Zhang et al., 2017). Thus, the microbial community richness and diversity increased, which enhanced community stability for maintaining high containments removal efficiency and resisting perturbations. A similar finding was observed in a previous study, which showed that the richness and diversity of anaerobic microbial communities were promoted by were observed, though most core bacterial genera were shared by the two sludge samples (Fig. 4). Thiobacillus had been detected as the primary and universal genus in coking wastewater treatment plants (Ma, Qu et al., 2015), and its relative abundance rose from 5.22\% in MBR up to $6.32 \%$ in MEBR. Comamonas was considered as a versatile aromatic degrader for PAHs and NHCs as well as a promising denitrifier and nitrifier under various aeration conditions (Ibarbalz et al., 2013; Jia et al., 2016). Abundance of Comamonas in MBR and MEBR were $5.02 \%$ and $6.28 \%$ respectively. Thauera, as a degrader for phenol, methylphenol and indole, increase from $1.53 \%$ to $2.61 \%$. Truepera was reported in close relation with the 
biodegradation of indole, and it abundance increased from $2.24 \%$ to $3.38 \%$, which was consistent with the indole removal. The abundance increment of these microbes might be the primary reason in term of microbial community for the enhancement of pollutants removals in MEBR. Moreover, the relative abundance of some genera for nitrification and denitrification or participated in nitrogen removal (eg. Nitrospira, Denitratisoma, Thermomonas) rose in varying degrees, which was mainly attributed to the anoxic/anaerobic microenvironment created by the large and compact sludge flocs. This indicated that MEBR had enhanced potential for nitrogen removal at controlled conditions.

\subsection{The relationships analyses}

The treatment performance enhancement, membrane fouling reduction, changes in the properties of the mixed liquor and microbial community evolution were all resulted from the addition of intermittent DC. The relationships analyses were conducted with ordination method to discern the effects of operational parameters. The relationship between microbial community structure and main operational parameters (electrical exposure mode, current density and MLSS) was illustrated in Fig. 5a. It could be inferred that current density and electrical exposure mode affected the microbial community significantly and MLSS influenced some of the genera. An obtuse angle between current density and exposure mode indicated that they had an adversely interactive impact in regulating microbial community compositions, since current density and exposure mode affected electrical amount in opposite direction. Thiobacillus, as the primary genus in coking wastewater treatment plants was affected by electrical exposure mode dramatically. The dynamics of Thauera and Comamonas were highly associated with current density and exposure mode. Variation of exposure mode exhibited a positive impact on variations of Thiobacillus, Nitrospira, Denitratisoma and 
Thermomonas. Negative correlations emerged between MLSS, current density and Azoarcus, GP4 and Isosphaera. The fact that microbial community was positively or negatively affected by the operational parameters might be a primary reason for the community shifts. The correspondence analysis between mixed liquor properties and operational parameters suggested that the changes of properties in mixed liquor were highly related with current density and electrical exposure mode (Fig. 5b). Current density and exposure mode exerted different impact on the variations of mixed liquor properties. Zeta potential was associated with electrical exposure mode significantly, since exposure mode determined the amount of positive charge iron ion in the long period operation, thus exerting considerable effect on zeta potential. SMP was influenced by current density with a high significance. PSD and SRF were related with both current density and exposure mode. Membrane fouling depended on the properties of the mixed liquor(Kurita et al., 2016; Abass et al., 2018). A regression model was gained between membrane fouling rate expressed by TMP increment over time and properties of mixed liquor with a high correlation coefficient (Formula $\mathrm{S} 2, R^{2}=0.95$ ), existed among microbial community structure, mixed liquor properties and operational parameters. A thorough and comprehensive understanding of the relationships would facilitate the optimizing control for fouling reduction as well as efficiency enhancement and be conducive to the rapid development of MEBR in domestic and industrial wastewater treatment. 
According to the above results, microbial community evolution in a long period of operation played a vital role in the performance enhancement of MEBR. It assumed that the treatment efficiency of MEBR might be well even as the intermittent DC was cut off. The treatment performance of MEBR with cutting off DC was investigated (Fig. 6). Average COD and total phenols removal efficiencies of MEBR decreased from $83.38 \%$ to $78.33 \%$ and 93.97\% to $87.97 \%$ respectively when intermittent DC was cut off. Treatment efficiency of MEBR when DC was cut off was still higher than the sum efficiencies of MBR and the electro-catalytic degradation $(71.3 \%+2.38 \%=73.68$ for $\mathrm{COD}, 82.47 \%+3.78 \%=86.25 \%$ for total phenols), which further confirmed the synergic effect between biodegradation and electrochemical effect in MEBR. The results suggested that the functional bacteria enriched by intermittent electric field still exerted dominating effect on pollutants removals, even though the DC was cut off. When the intermittent DC was switched on after a period of cutting off, COD and total phenols removal efficiencies recovered gradually in 4 days, verifying the comprehensive effects of mixed liquor properties and microbial community succession induced by intermittent DC with iron anode on the enhancement of treatment performance. The variations of membrane fouling were also investigated by monitoring the TMP. When intermittent DC was cut off, TMP increment rate of MEBR increased, indicating the exacerbation of membrane fouling without the assistance of electrokinetic effects. However, membrane fouling rate of MEBR $(0.42 \mathrm{kPa} / \mathrm{d})$ was still lower than MBR $(0.56$ $\mathrm{kPa} / \mathrm{d}$ ) when DC was cut off (Fig. S3), resulting from the residual electrokinetic effects. The exacerbation of membrane fouling of MEBR as cutting off DC was mainly associated with the changes in the mixed liquor. When the intermittent DC was switched on again, membrane fouling was hindered accordingly. The long term operation of MEBR with high efficiency 
also demonstrated the treatment stability and membrane fouling migration of MEBR with intermittent DC.

The study demonstrated that MEBR enhanced the treatment performance and substantially hindered membrane fouling with the employment of DC and electrodes. The analyses of the mechanism and relationships were beneficial to the operation and optimization of MEBR. The net cost increment of MEBR was mainly associated with the DC energy demand and sacrificial anode, which were related to the raw wastewater properties, operating conditions such as current density, exposure mode and HRT as well as the electrode price. The DC energy demand and anode consumption of MEBR were calculated to be 0.036 needed to be considered. 
MEBR with iron anode at appropriate exposure mode was found to be an efficient

method for hindering membrane fouling and promoting treatment performance, primarily associating with the changes in properties of mixed liquor, such as the increase of floc size and reduction of SMP and SRF, caused by electrokinetic effects. Microbial activity was provoked to be enhanced by suitable current density and exposure. The microbial community evolution promoted by DC exposure also accounted for performance enhancement in MEBR. The understanding of relationships among microbial community structure, mixed liquor properties and operational parameters was beneficial to applying efficient strategies to MEBR.

\section{Acknowledgements}

This work was supported by the National Natural Science Foundation of China (NO. 51704114) and Hunan provincial Natural Science Foundation of China (NO. 2018JJ3175).

\section{References}

[1] Abass, O. K., Fang, F., Zhuo, M., Zhang, K., 2018. Integrated interrogation of causes of membrane fouling in a pilot-scale anoxic-oxic membrane bioreactor treating oil refinery wastewater. Sci. Total Environ. 642, 77-89.

[2] Ahmed, F. E., Hilal, N., Hashaikeh, R., 2018. Electrically conductive membranes for in situ fouling detection in membrane distillation using impedance spectroscopy. J. Membrane Sci. 556, 66-72.

[3] Bani-Melhem, K., Elektorowicz, M., 2010. Development of a novel submerged membrane electro-bioreactor (SMEBR): performance for fouling reduction. Environ. Sci. Technol. 44, 3298-3304.

[4] Bani-Melhem, K., Elektorowicz, M., 2011. Performance of the submerged membrane electro-bioreactor (SMEBR) with iron electrodes for wastewater treatment and fouling reduction. J. Membrane Sci. 379(1), 434-439.

[5] Barreto, C. M., Garcia, H. A., Hooijmans, C. M., Herrera, A., Brdjanovic, D., 2017. 
Assessing the performance of an MBR operated at high biomass concentrations. Int. Biodeter. Biodegr. 119(Supplement C), 528-537.

[6] Chen, M., Zhang, X., Wang, Z., Wang, L., Wu, Z., 2017. QAC modified PVDF membranes: Antibiofouling performance, mechanisms, and effects on microbial communities in an MBR treating municipal wastewater. Water Res. 120(Supplement C), 256-264.

[7] Dubois, M., Gilles, K. A., Hamilton, J. K., Rebers, P., Smith, F., 1956. Colorimetric method for determination of sugars and related substances. Anal. Chem. 28, 350-356.

[8] Fan, H., Xiao, K., Mu, S., Zhou, Y., Ma, J., Wang, X., Huang, X., 2018. Impact of membrane pore morphology on multi-cycle fouling and cleaning of hydrophobic and hydrophilic membranes during MBR operation. J. Membrane Sci. 556, 312-320.

[9] Hou, B., Han, H., Jia, S., Zhuang, H., Zhao, Q., Xu, P., 2014. Effect of alkalinity on nitrite accumulation in treatment of coal chemical industry wastewater using moving bed biofilm reactor. J. Environ. Sci-China 26(5), 1014-1022.

[10] Hou, B., Han, H., Zhuang, H., Xu, P., Jia, S., Li, K., 2015. A novel integration of three-dimensional electro-Fenton and biological activated carbon and its application in the advanced treatment of biologically pretreated Lurgi coal gasification wastewater. Bioresour. Technol. 196, 721-725.

[11] Huang, L., Lee, D.-J., 2015. Membrane bioreactor: A mini review on recent R\&D works. Bioresour. Technol. 194, 383-388.

[12] Ibarbalz, F. M., Figuerola, E. L. M., Erijman, L., 2013. Industrial activated sludge exhibit unique bacterial community composition at high taxonomic ranks. Water Res. 47(11), 3854-3864.

[13] Ibeid, S., Elektorowicz, M., Oleszkiewicz, J. A., 2013. Novel electrokinetic approach reduces membrane fouling. Water Res. 47(16), 6358-6366.

[14] Ibeid, S., Elektorowicz, M., Oleszkiewicz, J. A., 2015. Electro-conditioning of activated sludge in a membrane electro-bioreactor for improved dewatering and reduced membrane fouling. J. Membrane Sci. 494, 136-142.

[15] Iorhemen, O. T., Hamza, R. A., Tay, J. H., 2017. Membrane fouling control in membrane bioreactors (MBRs) using granular materials. Bioresour. Technol. 240(Supplement C), 
9-24.

[16] Jia, S., Han, H., Hou, B., Zhuang, H., Fang, F., Zhao, Q., 2014. Treatment of coal gasification wastewater by membrane bioreactor hybrid powdered activated carbon (MBR-PAC) system. Chemosphere 117, 753-759.

[17] Jia, S., Han, H., Zhuang, H., Hou, B., 2016. The pollutants removal and bacterial community dynamics relationship within a full-scale British Gas/Lurgi coal gasification wastewater treatment using a novel system. Bioresour. Technol. 200, 103-110.

[18] Jiang, B., Du, C., Shi, S., Tan, L., Li, M., Liu, J., Xue, L., Ji, X., 2017. Enhanced treatment performance of coking wastewater and reduced membrane fouling using a novel EMBR. Bioresour. Technol. 229, 39-45.

[19] Kurita, T., Mogi, T., Kimura, K., 2016. Influence of different biofilm carriers on the operation and membrane fouling of submerged membrane bioreactors. Sep. Purif. Technol. 169, 43-49.

[20] Liu, J., Xiong, J., Tian, C., Gao, B., Wang, L., Jia, X., 2018. The degradation of methyl orange and membrane fouling behavior in anaerobic baffled membrane bioreactor. Chem. Eng. J. 338, 719-725.

[21] Liu, L., Liu, J., Gao, B., Yang, F., 2012. Minute electric field reduced membrane fouling and improved performance of membrane bioreactor. Sep. Purif. Technol. 86, 106-112.

[22] Lowry, O. H., Rosebrough, N. J., Farr, A. L., Randall, R. J., 1951. Protein measurement with the Folin phenol reagent. J. Biol. Chem. 193, 265-275.

[23] Ma, Q., Qu, Y., Shen, W., Zhang, Z., Wang, J., Liu, Z., Li, D., Li, H., Zhou, J., 2015. Bacterial community compositions of coking wastewater treatment plants in steel industry revealed by Illumina high-throughput sequencing. Bioresour. Technol. 179(Supplement C), 436-443.

[24] Martin Garcia, I., Mokosch, M., Soares, A., Pidou, M., Jefferson, B., 2013. Impact on reactor configuration on the performance of anaerobic MBRs: Treatment of settled sewage in temperate climates. Water Res. 47(14), 4853-4860.

[25] Meng, F., Zhang, S., Oh, Y., Zhou, Z., Shin, H.-S., Chae, S.-R., 2017. Fouling in membrane bioreactors: An updated review. Water Res. 114, 151-180.

[26] Ruigómez, I., González, E., Guerra, S., Rodríguez-Gómez, L. E., Vera, L., 2017. 
Evaluation of a novel physical cleaning strategy based on HF membrane rotation during the backwashing/relaxation phases for anaerobic submerged MBR. J. Membrane Sci. 526,

[27] Tafti, A. D., Seyyed Mirzaii, S. M., Andalibi, M. R., Vossoughi, M., 2015. Optimized 181-190. coupling of an intermittent DC electric field with a membrane bioreactor for enhanced effluent quality and hindered membrane fouling. Sep. Purif. Technol. 152(Supplement C), 7-13.

[28] Thrash, J. C., Coates, J. D., 2008. Review: direct and indirect electrical stimulation of microbial metabolism. Environ. Sci. Technol. 42, 3921-3931.

[29] Tian, Y., Chen, L., Zhang, S., Cao, C., Zhang, S., 2011. Correlating membrane fouling with sludge characteristics in membrane bioreactors: An especial interest in EPS and sludge morphology analysis. Bioresour. Technol. 102(19), 8820-8827.

[30] Xie, M., Shon, H. K., Gray, S. R., Elimelech, M., 2016. Membrane-based processes for wastewater nutrient recovery: Technology, challenges, and future direction. Water Res. $89,210-221$.

[31] Xin, X., He, J., Wang, Y., Feng, J., Qiu, W., 2016. Role of aeration intensity on performance and microbial community profiles in a sequencing batch reaction kettle (SBRK) for wastewater nutrients rapid removal. Bioresour. Technol. 201, 140-147.

[32] Xu, C., Han, H., Jia, S., Zhao, Q., 2016. Influence of phenol on ammonia removal in an intermittent aeration bioreactor treating biologically pretreated coal gasification wastewater. J. Environ. Sci-China 43(Supplement C), 99-105.

[33] Xu, P., Xu, H., Shi, Z., 2018. A novel bio-electro-Fenton process with FeVO4/CF cathode on advanced treatment of coal gasification wastewater. Sep. Purif. Technol. 194, 457-461.

[34] Zhang, J. X., Zhang, Y. B., Quan, X., Li, Y., Chen, S., Zhao, H. M., Wang, D., 2012. Ananaerobic reactor packed with a pair of Fe-graphite plate electrodes for bioaugmentation of azo dye wastewater treatment. Biochem. Eng. J. 63, 31-37.

[35] Zhang, S., Huang, Z., Lu, S., Zheng, J., Zhang, X., 2017. Nutrients removal and bacterial community structure for low $\mathrm{C} / \mathrm{N}$ municipal wastewater using a modified anaerobic/anoxic/oxic (mA2/O) process in North China. Bioresour. Technol. 243, 
489 
491 Table 1 Results of GC-MC analysis for the influent and effluents of MBR and MEBR

\begin{tabular}{|c|c|c|c|}
\hline Compounds & Raw & MBR & MEBR \\
\hline \multicolumn{4}{|l|}{ Phelols } \\
\hline Phenol & $10.9^{\mathrm{a}}$ & 0.8 & 0.5 \\
\hline Hydroquinone & 0.5 & 0.7 & 0.9 \\
\hline Cathchol & 1.3 & 0.9 & 0.9 \\
\hline P-Cresol & 0.4 & 0.2 & 0.3 \\
\hline Phenol,3-ethylphenol & 1.6 & ND & $\mathrm{ND}^{\mathrm{b}}$ \\
\hline Phenol,2,3-dimethyl & 0.6 & ND & ND \\
\hline Phenol,4-tert-butyl & 1.7 & 0.9 & 0.8 \\
\hline 1,3-Benzenediol, 4,5-dimethyl- & 1.6 & ND & ND \\
\hline Phenol,2,4-di-tert-butyl & 0.6 & ND & ND \\
\hline 2,6-Di-tert-butyl-4-methylphenol & 1.5 & 0.9 & 0.6 \\
\hline 1,3-Benzenediol, 4,5-dimethyl- & 1.6 & 0.5 & 0.5 \\
\hline Phenol,4-methyl-2-nitro & 1.2 & 0.6 & 0.5 \\
\hline Phenol,4-bis(1,1-dimethylethyl)- & 0.7 & 0.2 & ND \\
\hline Phenol,2-[(trimethylsilyl)oxy] & ND & 0.8 & 0.9 \\
\hline Phenol,2,6-dimethyl-4-nitro & ND & 0.2 & 0.2 \\
\hline
\end{tabular}

Nitrogenous heterocyclic compounds

Quinoline

Isoquinoline
2.6

2.4
1.3

0.9

1.5 
6-Amino-2-methyl quinoline

1.6

0.8

0.5

Pyridine

2.1

1.7

1.6

Pyridine,3-methyl-

1.9

1.3

1.1

2-Amino-4-methyl pyrimidine

1.2

0.8

0.7

Indole

1.4

0.2

ND

Indole, 2- methyl

1.1

ND

ND

2-Amino-3-picoline

1.6

0.7

0.6

2- methyl oxindole

1.4

0.3

ND

2,7-Diphenyl-1H-indole

1.2

ND

ND

2-Pyridine-carboxaldehyde

ND

0.6

0.6

3-Pyridine-carboxaldehyde

ND

0.8

0.5

1H-imidazole, 1-methyl-2-nitro-

1.1

0.8

0.6

5-Methyl-2-nitro-1H-imidazole

1.2

0.3

ND

Long-chain hydrocarbons

Undecane

Dodecane

1.2

ND

ND

Hexadecane

0.9

0.6

0.5

Heptadecane

1.2

0.8

0.6

Octadecane

1.1

0.3

ND

Eicosane

0.8

ND

ND

Heneicosanoic

0.6

ND

ND

Docosanoic

1.7

1.2

0.9 


\begin{tabular}{|c|c|c|c|}
\hline Pentacosane & 0.8 & 0.5 & 0.3 \\
\hline Hexacosane & 0.9 & 0.6 & 0.5 \\
\hline Octacosane & 0.5 & 0.3 & 0.3 \\
\hline Nonacosane & ND & 0.1 & 0.1 \\
\hline \multicolumn{4}{|l|}{ Polynuclear aromatic hydrocarbons } \\
\hline Naphthalene & 1.9 & 1.2 & 0.8 \\
\hline Naphthalene,1,3-dimethyl & 1.5 & 1.1 & 0.9 \\
\hline Naphthalene, 2,6-Dimethyl & 1.6 & 1.2 & 0.9 \\
\hline Naphthalene, 1,2,6-Trimethyl & 0.2 & ND & $\mathrm{ND}$ \\
\hline Naphthalene, 1,2,6,7-Tetramethyl & 0.2 & 0.2 & 0.3 \\
\hline \multicolumn{4}{|l|}{ Esters } \\
\hline Dimethyl phthalate & 1.2 & 0.7 & 0.6 \\
\hline Diisobutyl phthalate & 0.8 & 0.6 & 0.4 \\
\hline Dibutyl phthalate & 1.2 & 0.3 & ND \\
\hline Hexanedioic acid, bis(2-ethylhexyl) ester & 0.5 & ND & ND \\
\hline Sum & 65.9 & 28.8 & 22.3 \\
\hline
\end{tabular}

a. Values represent the relative percentage of total peak area.

b. ND, not detected.

494 


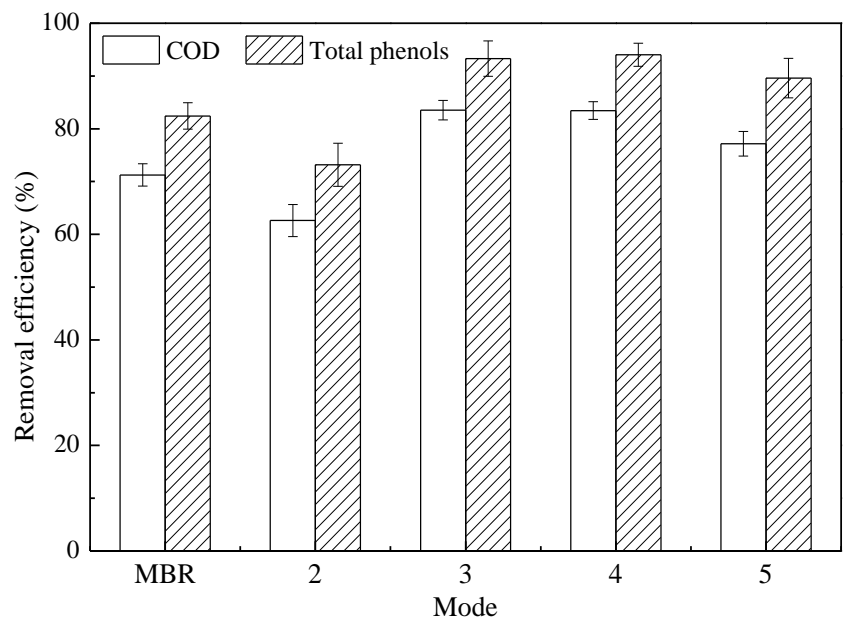

498 Fig. 1 Performances of MBR and MEBR at various electrical exposure modes (mode 2:

499 20'-OFF/10'-ON, mode 3: 21.5'-OFF/7.5'-ON, mode 4: 24'-OFF/6'-ON, mode 5: $\left.500 \quad 25^{\prime}-\mathrm{OFF} / 5^{\prime}-\mathrm{ON}\right)$

501

502 

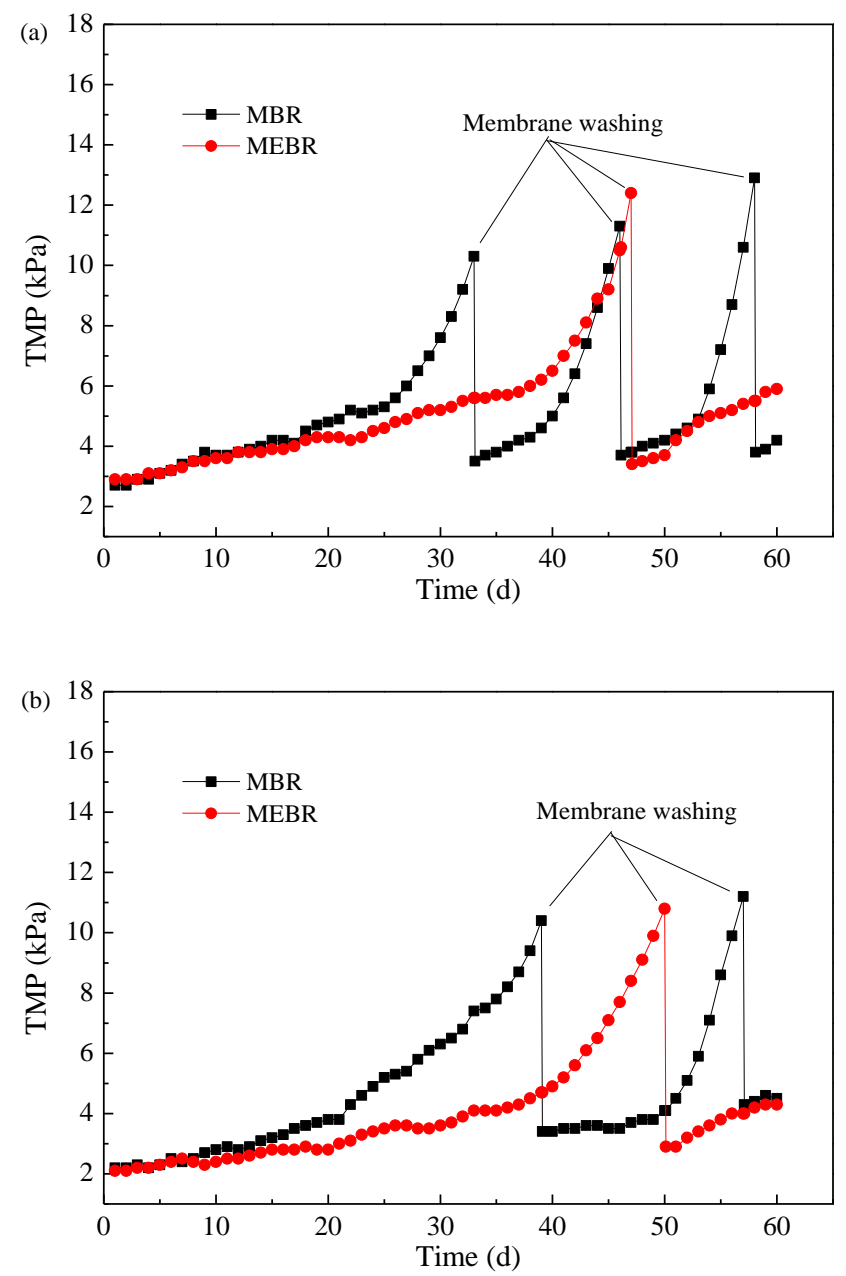

505

Fig. 2 Variation of TMP in MBR and MEBR (a. MLSS 4500 mg/L, b. MLSS 3000 mg/L) 

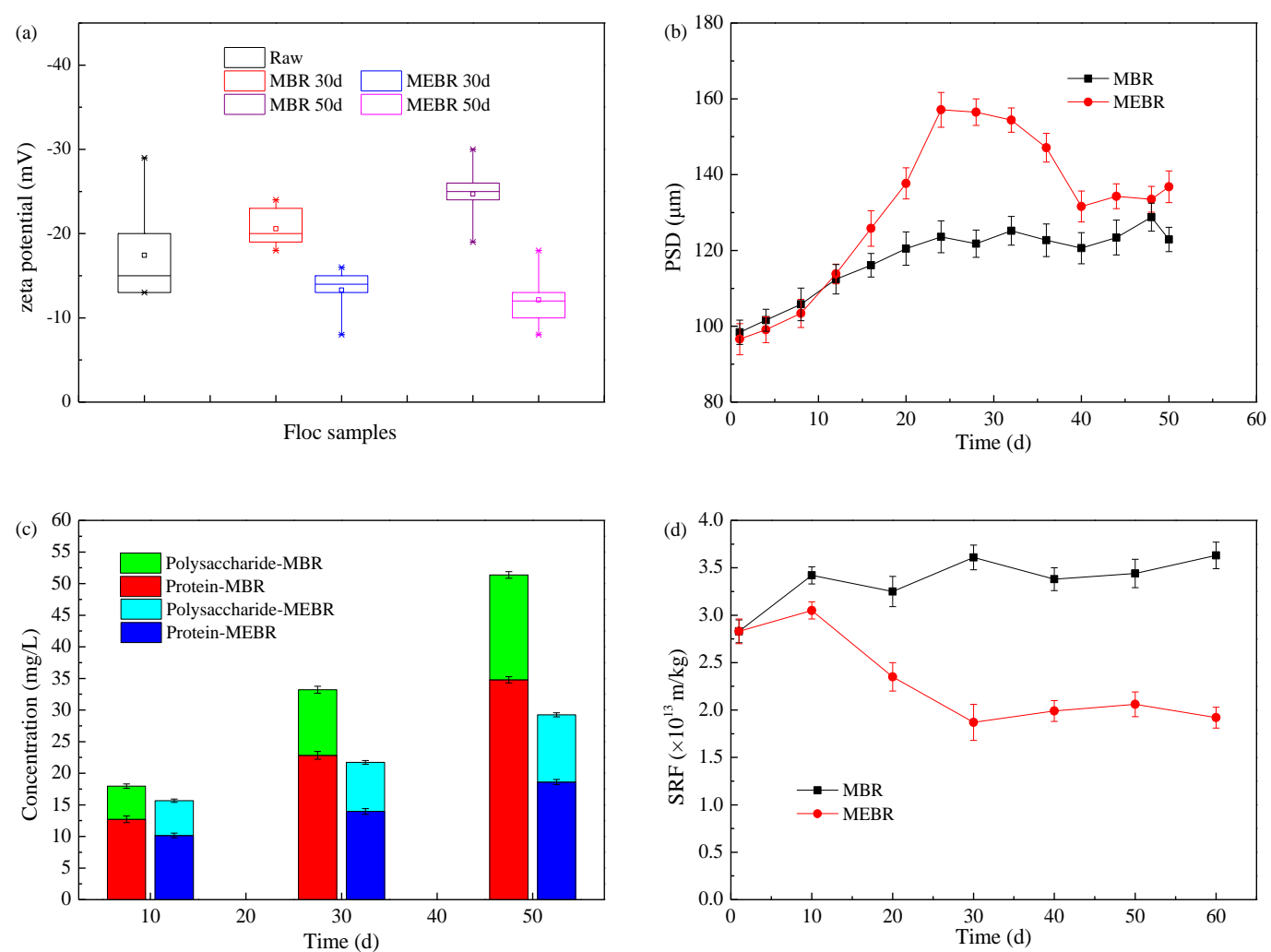

511

Fig. 3 Changes of mixed liquor properties in MBR and MEBR (a. zeta potential, b. floc PSD, c. SMP, d. SRF)

514 


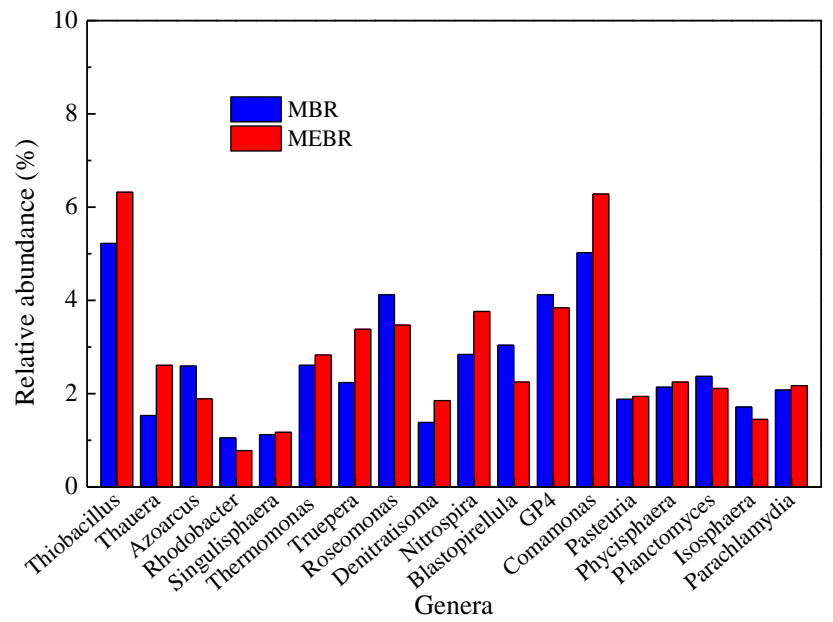

519 Fig. 4 Evolution of the primary genera in MBR and MEBR

520

521 


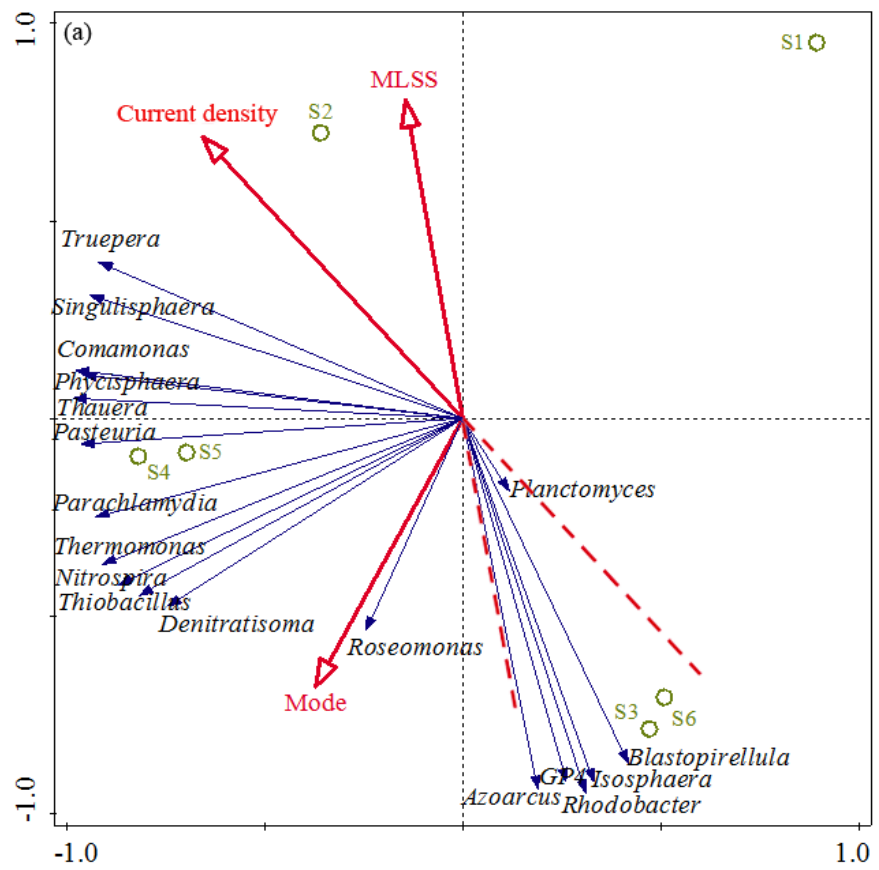

523

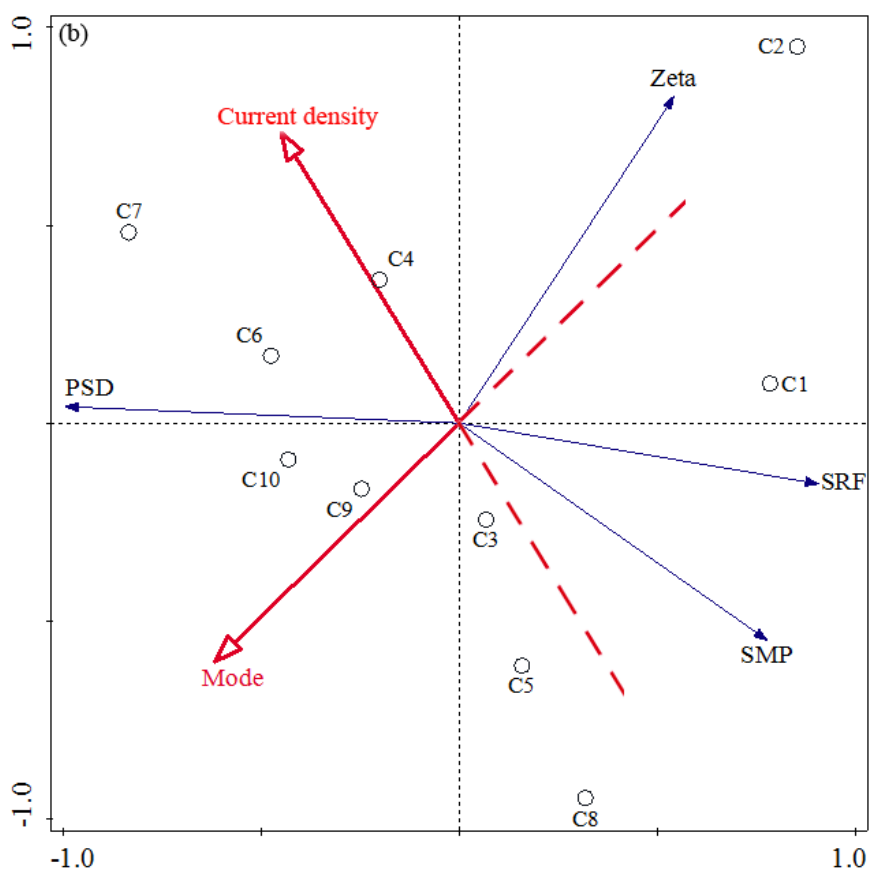

524

525

526 527

Fig. 5 Principal component analysis (PCA) of the correlations (a. microbial community composition vs operational parameters, b. mixed liquor properties vs operational parameters) 


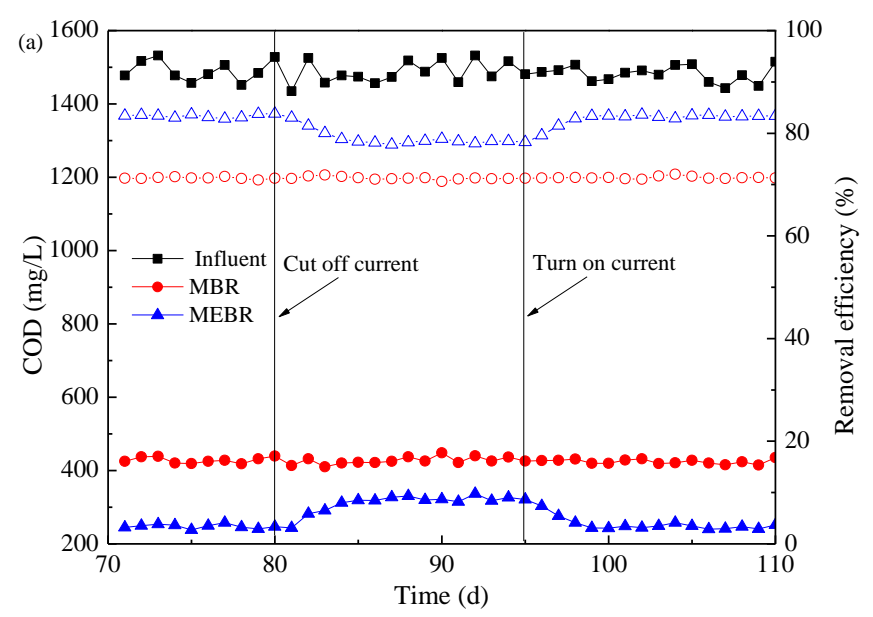

530

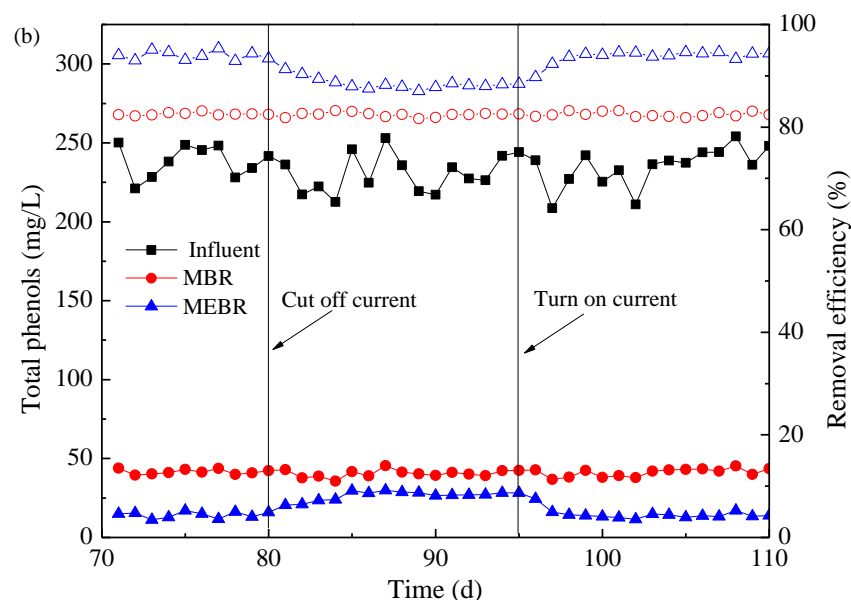

Fig. 6 Long-term treatment performance of MEBR (a. COD, b. total phenols) 


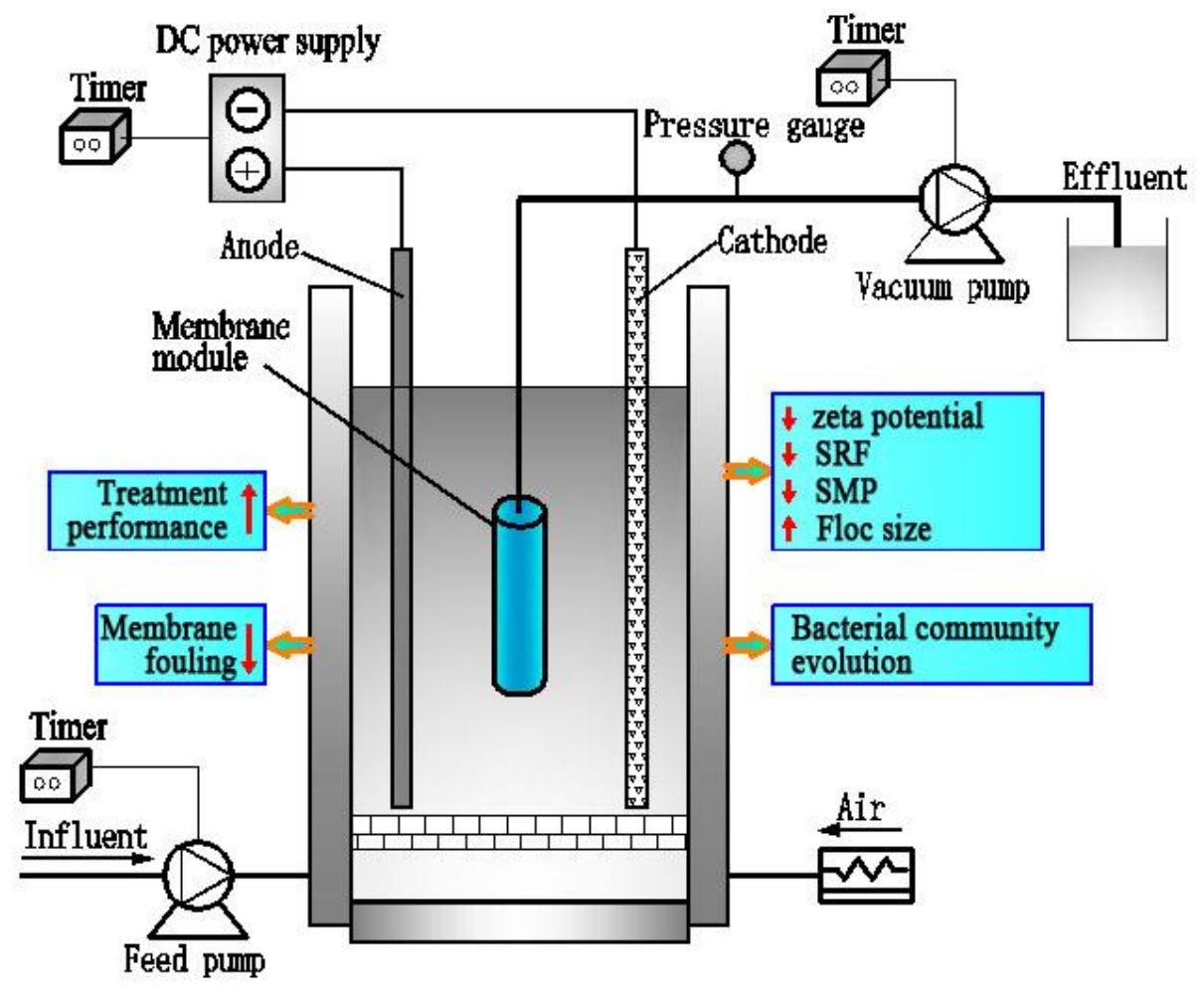

Highlights

MEBR coupling intermittent DC and MBR was established to treat coal chemical industry

wastewater.

- MEBR exhibited significant efficiency improvement and membrane fouling reduction.

Changes in properties of mixed liquor caused by electrokinetics accounted for fouling

544 migration.

MEBR with intermittent DC and iron anode promoted microbial community evolution. 